\title{
LA CONSTRUCCIÓN DEL RÉGIMEN JURÍDICO DEL CLIMA ENTRE CIENCIA, DERECHO Y POLÍTICA ECONÓMICA
}

\section{FORMULATING THE CLIMATE LEGAL SYSTEM: SCIENCE, LAW AND ECONOMIC POLICY}

\author{
MARTA TORRE-SchaUB \\ Directora-catedrática de investigación en el Centro Nacional de la Investigación \\ Científica (CNRS) y \\ del Instituto de Ciencia Jurídica y Filosófica de la Sorbona (ISJPS) \\ de la Universidad París 1 Panthéon-Sorbonne \\ Directora y fundadora de la red internacional de investigadores ClimaLex \\ (Derecho y Cambio Climático) \\ marthe.torre-schaub@univ-paris1.fr
}

Fecha de recepción: 30 de abril de 2019 / Fecha de aceptación: 18 de junio de 2019

\begin{abstract}
Resumen: El derecho relativo al cambio climático es el producto de la reacción al grito de alarma de los científicos sobre la degradación irreversible del clima. El derecho del clima no habría podido desarrollarse de no haber sido por estas alertas lanzadas por la comunidad científica. Las políticas climáticas, a su vez, son igualmente dependientes del estado de la ciencia y de los expertos científicos. El vínculo entre la actividad humana y su influencia en la estabilidad del clima global se estableció primeramente a nivel científico y después fue llevado ante la comunidad internacional. La construcción del derecho del clima es, pues, producto de diferentes factores y situaciones. Por una parte, el derecho del clima ha debido integrar las informaciones científicas referentes a los procesos ecológicos. En segundo lugar, el derecho del clima se ha desarrollado en una dinámica de derecho internacional ya existente, al tiempo que ha creado nuevas instituciones. En tercer lugar, el derecho relativo al clima se ha formado igualmente a partir de diversos elementos e instrumentos. De esta forma, el cambio climático ha dado lugar a un derecho que presenta unas características marcadas por la mezcla de ingredientes, así como por su carácter altamente evolutivo. Este artículo tiene por objeto analizar estos diferentes elementos y evoluciones hasta la formación del derecho climático actual.
\end{abstract}


Abstract: Climate change law is the product of scientists' reaction to the warning cry of irreversible degradation of the climate. Climate law could not have developed had it not been for the warnings issued by the scientific community. Climate policies, in turn, are equally dependent on the state of the science and scientific experts. The link between human activity and its influence on global climate stability was first established at the scientific level and subsequently brought before the international community. The formulation of climate law is therefore the product of different factors and situations. First of all, climate law has had to integrate scientific information on ecological processes. Secondly, climate law has developed within the existing context of international law while at the same time creating new institutions. Thirdly, climate law has also been built upon the foundation of a wide range of elements and instruments. Thus, climate change has given rise to a legal system with characteristics shaped by the combination of these ingredients, as well as by its highly evolutionary nature. The aim of this article is to examine the different elements and evolutions that have led to the formation of current climate law.

Resum: El dret relatiu al canvi climàtic és el producte de la reacció al crit d'alarma dels científics sobre la degradació irreversible del clima. El dret del clima no hauria pogut desenvolupar-se sense les alertes Ilançades per la comunitat científica. Les polítiques climàtiques, al seu torn, són igualment dependents de l'estat de la ciència i dels experts científics. El vincle entre l'activitat humana i la seva influència en l'estabilitat del clima global es va establir primerament en l'àmbit científic i després va ser portat davant la comunitat internacional. La construcció del dret del clima és, doncs, producte de diferents factors i situacions. D'una banda, el dret del clima ha hagut d'integrar les informacions científiques referents als processos ecològics. En segon lloc, el dret del clima s'ha desenvolupat en una dinàmica de dret internacional ja existent, al mateix temps que ha creat noves institucions. En tercer lloc, el dret relatiu al clima s'ha format igualment a partir de diversos elements i instruments. D'aquesta forma, el canvi climàtic ha donat lloc a un dret que presenta unes característiques marcades per la barreja d'ingredients, així com pel seu caràcter altament evolutiu. Aquest article té per objecte analitzar aquests diferents elements $i$ evolucions fins a la formació del dret climàtic actual.

Palabras clave: Cambio climático - Derecho climático - Justicia climática Informes científicos - Expertos del clima - Convención Marco de las Naciones Unidas - Acuerdo de París - Irreversibilidad - Incertidumbre - Límites planetarios - Clima estable

Key Words: Climate Change - Climate Change Law - Climate Justice - Scientific expertise - Climate Science - United Nations Convention-framework - Paris Agreement - irreversibility - uncertainty - Planetary Boundaries - liveable Climate 
Paraules clau; Canvi climàtic - Dret climàtic - Justícia climática - Informes científics - Experts del clima - Convenció Marc de les Nacions Unides - Acord de París - Irreversibilitat - Incertesa - Límits planetaris - Clima estable

SUMARIO: I. Introducción. II. El diálogo entre ciencia y derecho, una lógica de impulso. 1. La puesta en marcha de un mecanismo de colaboración. 2. "Estabilizar" el clima: una idea de vital importancia. 3. La epistemología científica del IPCC como método de consolidación normativa. 4. Lenguaje científico y lenguaje jurídico: ¿una dinámica económica? 5. El Protocolo de Kioto: ¿dónde queda la "universalidad" de la lucha contra el cambio climático? III. El Acuerdo de París y la relación entre ciencia y derecho, ¿lost in translation? 1. Irreversibilidad, mitigación y adaptación, ¿qué instrumento jurídico? 2. Copenhague, ¿una línea discontinua en el diálogo entre ciencia y derecho? 3. "Siempre nos quedará París...": el Acuerdo de París, una continuidad aparente. IV. Conclusión. V. Bibliografía

\section{INTRODUCCIÓN}

En poco menos de cien años podremos ver París inundado por el Sena, las Maldivas y Tuvalu borrados del mapa, los inuits despojados de sus territorios y cultura; este escenario catastrófico no es solo el resultado de sombrías predicciones, sino también de hipótesis científicas sólida y debidamente probadas y argumentadas sobre la evolución del clima desde la segunda mitad del siglo pasado ${ }^{1}$. Numerosos estudios científicos elaborados por prestigiosas instituciones vienen presentando desde la década de 1970 el fenómeno del calentamiento global como algo serio y seguro. A pesar de estas advertencias, por motivos relacionados con las inercias de la comunidad internacional, presupuestarios y políticos, el IPCC no fue creado hasta 1988². El IPCC se estableció en noviembre de 1988 a instancias del G7 por dos organismos de las Naciones Unidas: la Organización Meteorológica Mundial (OMM) y el Programa de las Naciones Unidas para el Medio Ambiente (PNUMA) ${ }^{3}$. La decisión del G7 se tomó bajo la presión de Ronald Reagan y Margaret Thatcher para evitar que una agencia de las Naciones Unidas, de la que se tenían sospechas de

\footnotetext{
${ }^{1}$ SINAI, A., "Le climat, otage des lobbies industriels", Le Monde diplomatique, febrero 2001, p. 14 ; Travaux du Groupe intergouvernamental d'experts sur l'évolution des climats (IPCC), sobre todo el de1995, Les Cahiers de Global Chance, núm. 7, julio 1996.

2 IPCC Intergovernmental Panel on Climate Change (Grupo Intergubernamental de Expertos sobre el Cambio Climático). A partir de aquí, IPCC. Este grupo, creado en 1988 como resultado de una iniciativa política de carácter internacional, depende de la Organización Meteorológica Mundial y del Programa de las Naciones Unidas para el Medio Ambiente, y tiene la misión de evaluar, sin prejuicios, metódica, clara y objetivamente, la información científica, técnica y socioeconómica que necesitamos para comprender mejor los riesgos asociados con el calentamiento global de origen humano, para identificar con mayor precisión las posibles consecuencias de este cambio y para considerar posibles estrategias de adaptación y mitigación. No tiene el mandato de realizar investigaciones o monitorear cambios en las variables climáticas $u$ otros parámetros relevantes.

3 TORRE-SCHAUB, M., "La protection juridique du climat, entre gestion contractuelle et négociation économique", Revista Themis, Revista da Facultade de Direito da UNL, IV-núm. 6, 2003, pp. 47-41.
} 
cierto activismo ambiental, se hiciera cargo del análisis climático. Según Nigel Lawson, secretario de Energía y luego ministro de Hacienda en el Gobierno de Margaret Thatcher, su objetivo era contrarrestar la influencia de los sindicatos de mineros de carbón mediante el apoyo a la energía nuclear como fuente de energía limpia para reemplazar al carbón. Pese a este trasfondo político confuso y poco claro, los primeros trabajos de los expertos científicos del grupo permitieron crear una dinámica positiva que ha alimentado desde entonces las negociaciones de la ONU en materia climática y con ello la redacción de los primeros textos jurídicos sobre el cambio climático.

En treinta años, entre 1988 y 2018, el IPCC ha emitido varios informes de evaluación, incluido el segundo informe de evaluación, publicado en 1995, que proporcionó a los negociadores documentos importantes antes de la adopción del Protocolo de Kioto en 1997. El tercer informe de evaluación se publicó en el 2001, el cuarto en el 2007 y el quinto en el 2014, justo antes de la COP 21 en París y de la redacción del Acuerdo de París.

El derecho relativo al cambio climático es así el producto de una reacción al grito de alarma de los científicos sobre la degradación irreversible del clima y sobre las terribles consecuencias de dicho fenómeno en el sistema de vida de nuestro planeta. Podemos afirmar que el derecho del clima no habría podido desarrollarse de no haber sido por las advertencias lanzadas por la comunidad científica ${ }^{4}$. Las políticas climáticas - a su vez basadas en este derecho- son igualmente dependientes del estado de la ciencia y de los expertos científicos ${ }^{5}$.

La influencia de la actividad humana en la estabilidad del clima global se demostró en primer lugar a nivel científico y después se presentó ante la comunidad internacional. Posteriormente, las Naciones Unidas asumieron el reto y adoptaron una serie de convenios internacionales a partir de la Cumbre de la Tierra, que tuvo lugar en Río de Janeiro en 1992. La relación entre la comunidad científica y la

\footnotetext{
4 JOUZEL, J., "La pluridisciplinarité au cœur du problème ", Torre-Schaub, M. (dir.), Dossier Spécial Droit et Climat, Les Cahiers Droit, Sciences \& Technologies, núm. 2, 2009, pp. 19-29.

5 TORRE-SCHAUB, M., "Le réchauffement climatique, une question interdisciplinaire", Torre-Schaub (dir.), Dossier Spécial Droit... cit., pp. 13-19.
} 
diplomacia internacional quedó bien establecida desde ese momento y ese mismo año se firmó la primera Convención Marco sobre el Cambio Climático ${ }^{6}$.

Pese al importante vínculo entre los avances científicos en materia climática y la producción de textos jurídicos en el plano internacional, el derecho del cambio climático ha debido tener en cuenta la cuestión de las políticas de desarrollo e industrialización, centrales en materia medioambiental. A pesar de que introduce el principio de desarrollo sostenible —cuya finalidad es la de compatibilizar el desarrollo económico e industrial con la protección del medio ambiente-, el derecho internacional del cambio climático refleja la forzosa cohabitación de varias dinámicas: la científica, la económica, la política y, por supuesto, la jurídica.

La construcción del derecho del clima es, pues, producto de varios factores combinados $^{7}$. En primer lugar, el derecho del clima ha debido integrar las informaciones científicas relativas a los procesos ecológicos, como el ciclo hidrológico, la atmósfera o la evolución del clima, a partir de los diferentes estudios y la literatura científica. En segundo lugar, el derecho del clima se ha desarrollado en una dinámica de derecho internacional ya existente, al tiempo que ha creado nuevas instituciones (las conferencias de las partes a la Convención Marco) y nuevas dinámicas (bottom up), reuniendo así nuevos actores y haciendo evolucionar de este modo las propias fuentes del derecho climático. En tercer lugar, y dado que la cuestión del cambio climático está estrechamente vinculada al desarrollo económico, industrial y energético de cada país y región del mundo, el derecho relativo al clima se ha formado igualmente a partir de elementos e instrumentos económicos (mercados de permisos de emisión, mecanismos de financiación de proyectos de desarrollo...). En consecuencia, el cambio climático ha dado lugar a un derecho que presenta unas características marcadas por la mezcla de ingredientes, así como por su carácter altamente evolutivo ${ }^{8}$.

\footnotetext{
${ }^{6}$ La Convención Marco de las Naciones Unidas sobre el Cambio Climático (CMNUCC) fue adoptada en Nueva York el 9 de mayo de 1992 y entró en vigor el 21 de marzo de 1994. Permite, entre otras cosas, reforzar la conciencia pública, a escala mundial, de los problemas relacionados con el cambio climático. En 1997, los Gobiernos acordaron incorporar una adición al tratado, conocida con el nombre de Protocolo de Kioto, que dispone medidas más enérgicas y jurídicamente vinculantes. En el 2006 se enmendó en Nairobi este Protocolo a la Convención Marco de las Naciones Unidas sobre Cambio Climático y se tenía previsto adoptar un nuevo protocolo en el año 2009 en Copenhague.

7 BOISSON DE CHAZOURNES, L., "Le droit et l'universalité de la lutte contre le changement climatique", Torre-Schaub, (dir.), Dossier Spécial... cit., pp. 29-37.

8 Véase TORRE-SCHAUB, M., "La construction juridique d'un droit à un climat stable : le droit autour de $2^{\circ} \mathrm{C}$ ", Torre-Schaub, M. et al. (dirs.), Normes et Changement climatique, regards interdisciplinaires,
} 
Llegados a este punto, cabe preguntarse hasta qué punto el derecho del clima ha sabido (o podido) desarrollar los contenidos científicos. ¿Qué valor científico posee el derecho del clima? ¿Y cómo traduce este último las informaciones científicas? Siguiendo un análisis que postula el llamado "pluralismo de verdades", este artículo sostiene que el paso de las informaciones científicas a su plasmación en un texto de naturaleza jurídica no solo no es automático, sino que es el producto de una elaboración delicada y a veces sofisticada. No planteamos aquí, pues, ni el "todo científico" ni el "todo jurídico", sino que nos interesaremos precisamente en la operación que permite el paso de uno al otro, siendo este proceso en sí mismo digno de estudio y consideración. En efecto, el derecho del clima, tal y como se ha venido construyendo desde hace treinta años, no se limita a incorporar las informaciones científicas ni a traducir pura y simplemente las reglas del derecho internacional del medio ambiente, sino que posee carácter propio. El derecho del cambio climático, tal y como se ha venido elaborando en los últimos treinta años, supone una serie de principios, derechos y técnicas que lo convierten en uno de los sectores del derecho más innovadores ${ }^{10}$. Así pues, en estas páginas se intentará analizar la manera en la que las "verdades" científicas y las "técnicas" jurídicas se entrelazan hasta formar el actual derecho climático.

Para una mejor comprensión de estas cuestiones y con el propósito de encontrar algunas respuestas, no nos conformaremos aquí con una descripción lineal y meramente cronológica de la formación del derecho del clima, sino que emplearemos una metodología en estratos. El derecho del cambio climático se ha formado en diversas etapas, cada una de ellas marcadas por un factor esencial que nos servirá de hilo conductor: el de las diversas fases de la relación entre los avances científicos en materia climática y su plasmación en términos jurídicos. A su vez, cada avance normativo ha venido acompañado de instrumentos y técnicas jurídicas distintas. En efecto, el derecho del cambio climático tuvo en sus inicios un carácter muy general y poco obligatorio, luego conoció una etapa caracterizada por una mayor fuerza obligatoria, si bien con un marcado énfasis económico, para

Actes du Colloque du 9 novembre 2018, Lancement du GDR ClimaLex, Mare et Martin, París, 2019, pp. 42-65 (en imprenta).

${ }_{9}$ En este sentido, véanse los análisis de NAIM-GISBERT, E., Les dimensions scientifiques du droit de l'environnement, Contribution à l'étude des rapports science-droit, Bruylant, 1999.

10 TORRE-SCHAUB, M., "Vers un nouveau paradigme socio-environnemental dans la gouvernance du climat", Torre-Schaub, M., Lavorel, S. y Moliner-Dubost, M. (dirs.), Quel(s) droit(s) pour les changements climatiques?, Mare et Martin, París, 2018, pp. 35-55. 
actualmente hallarse en una etapa de compromisos voluntarios, pero con instrumentos más variados y más adaptados a la evolución de la comunidad internacional y de la sociedad civil.

Este artículo se divide así en dos partes; cada una de ellas aborda y analiza las distintas etapas por las que ha pasado el derecho del cambio climático desde sus orígenes hasta la actualidad. Veremos así las influencias y confluencias de la ciencia del cambio climático en el derecho, y las incidencias y los avatares de los distintos instrumentos (económicos, obligatorios, de cooperación, voluntarios) elegidos en cada una de esas etapas. Esta visión panorámica nos permitirá poner en perspectiva la formación de un derecho relativamente joven y en plena evolución. Solo así podremos plantear algunas propuestas prospectivas sobre las futuras orientaciones de este derecho específico.

La primera parte aborda el diálogo entre ciencia y derecho como una dinamica positiva de impulso (1). La segunda parte analiza el riesgo actual de una dilatación o discontinuidad en la relación entre ciencia y derecho (2).

\section{EL DIÁLOGO ENTRE CIENCIA Y DERECHO, UNA LÓGICA DE IMPULSO}

Las negociaciones internacionales en materia climática respondían en sus inicios a una dinámica positiva. El climatólogo Jean Jouzel, que fue copresidente del grupo I del IPCC ${ }^{11}$, describe este proceso desde un enfoque histórico y sitúa sus orígenes en la década de 1970, cuando surgen las primeras advertencias sobre el calentamiento global y el aumento de la concentración de gases de efecto invernadero en la atmósfera.

\section{La puesta en marcha de un mecanismo de colaboración}

\footnotetext{
${ }^{11}$ EI IPCC está organizado en tres grupos de trabajo: el grupo I estudia los principios físicos del cambio climático; el grupo II, los impactos, la vulnerabilidad y la adaptación al cambio climático; el grupo III, las formas de mitigar el cambio climático ha publicado un informe especial en SRES, "Informe especial sobre escenarios de emisión", que ha sido la base de algunas simulaciones realizadas por los grupos de trabajo. Además, un equipo especial para los inventarios nacionales de gases de efecto invernadero ha elaborado guías para dichos inventarios. Cada grupo de trabajo (y el equipo de trabajo) tiene dos copresidentes, uno que representa a los países desarrollados y otro a los países en desarrollo.
} 
En las décadas de 1970 y 1980, la comunidad científica empieza a advertir del riesgo de calentamiento global a partir de estudios realizados sobre la base del enfoque de los modeladores del clima que informan sobre diversos grados de calentamiento durante el siglo XXI. El estudio del hielo polar (paleoclima) muestra el fuerte vínculo existente entre el efecto invernadero y la evolucion del clima a lo largo del tiempo y permite, entre otras cosas, disponer de datos suficientemente fiables como para avanzar la hipótesis más que plausible de un aumento anormal de temperaturas y de una concentración excesiva de gases de efecto invernadero en la atmósfera desde principios del siglo $X X X^{12}$.

Las advertencias se tienen en cuenta y, a finales de la década de 1980, se crea el IPCC gracias al impulso de científicos como Bert Bolin, que tenía una visión realista de las políticas a implantar. En efecto, cabe recordar que es el G7 el que impulsa el grupo a instancias de George Bush y de Margaret Thatcher. EI IPCC nació en 1988 $y$, a pesar de que hay que esperar cuatro años hasta el primer texto de naturaleza jurídica sobre el cambio climático - la Convención Marco de las Naciones Unidas de 1992 - la creación del grupo de expertos y su particular naturaleza permitirán un impulso político y jurídico mucho más importante que en otros campos del medio ambiente. Esto se debe a varias razones.

En primer lugar, quienes impulsaron este grupo de expertos comprendieron de inmediato que el fenómeno del calentamiento global debía analizarse de forma muy amplia, es decir, no solo el fenómeno en sí (las causas del calentamiento global), sino también sus consecuencias. $Y$ ya desde el inicio del IPCC se habló de adaptación y de las soluciones a implementar.

Gracias a esta visión tan amplia, los expertos del IPCC han ido ganando visibilidad. Ello se debe a que este grupo de expertos ha sido desde sus inicios puesto en marcha de forma muy pensada y atendiendo a reglas de funcionamiento epistemológico muy transparentes ${ }^{13}$. Su misión es clara: no plantear recomendaciones a los responsables de las políticas, sino limitarse a un diagnóstico. Este punto es esencial, ya que, por una parte, explica la independencia y fiabilidad

\footnotetext{
12 JOUZEL, J., "La pluridisciplinarité au cœur du problème", Torre-Schaub (dir.), Dossier Spécial... cit., p. 20 y ss.

${ }^{13}$ LECLERC, O., "Les règles de production des énoncés au sein du Groupe d'experts gouvernmental sur l'évolution du climat", Encinas de Munagorri, R. (dir.), Expertise et gouvernance du changement climatique, LGDJ, coll Droit et Société, París, 2009, pp. 59-89.
} 
de los expertos de este grupo, y, por otra, permite comprender la diferencia existente entre las conclusiones de los expertos del IPCC y lo que luego se plasma en los textos de derecho.

EI IPCC mantiene actualmente dicha línea, lo cual explica en gran parte también la situación actual de fuerte dualidad (por no decir esquizofrenia) entre la gravedad y urgencia plasmadas en los informes científicos y la lentitud política a la hora de adoptar medidas de atenuación del cambio climático más ambiciosas ${ }^{14}$.

La filosofía y misión del IPCC es realizar un diagnóstico, no plantear recomendaciones a los responsables políticos. El objetivo de la comunidad científica es dar a los responsables de las políticas los elementos necesarios para tomar sus decisiones. La relación entre la comunidad científica y la decisión política plasmada en los textos jurídicos responde a una situación de independencia y, al mismo tiempo, de dualidad. Por una parte, existen los diagnósticos de la comunidad científica y el IPCC, y, por otra, lo que luego se plasma en los textos. Nos referimos aquí a la Convención Marco, que se establece después del primer informe del IPCC.

El primer informe del IPCC es suficientemente claro, ya que prevé un calentamiento de la temperatura global de tres grados y un aumento del nivel del mar de 65 centímetros en la segunda mitad del siglo XXI. Aspectos que se reflejan en la Convención Marco ya en $1992^{15}$

Estas informaciones científicas son vitales e impulsan el proceso de elaboración de la Convención.

\footnotetext{
${ }^{14}$ DAHAN, A. y GUILLEMOT, H., Les relations entre science et politique dans le régime climatique : à la recherche d'un nouveau modèle d'expertise ?, Natures, Sciences, Sociétés, vol. 23, núm. Supplément, S6 - S 18, 2015; DAHAN, A., "La gouvernance climatique : entre climatisation du monde et schisme de réalité", L'Homme et la Société, núm. 199, pp. 79-90, julio 2016.

${ }^{15}$ We are certain of the following: there is a natural greenhouse effect...; emissions resulting from human activities are substantially increasing the atmospheric concentrations of the greenhouse gases: $\mathrm{CO}_{2}$, methane, CFCs and nitrous oxide. These increases will enhance the greenhouse effect, resulting on average in an additional warming of the Earth's surface. The main greenhouse gas, water vapour, will increase in response to global warming and further enhance it. Sintesis del informe destinado a los responsables gouvernamentales del primer Informe del IPCC 1990. "Preocupados por el hecho de que la actividad humana ha aumentado significativamente las concentraciones de gases de efecto invernadero en la atmosfera y que este aumento refuerza el efecto invernadero natural y que en promedio resultará en un calentamiento de la superficie adicional y de la atmosfera, del que es probable que sufran los ecosistemas naturales y la humanidad...", §2 del preambulo de la Convencion-marco 1992.
} 


\section{2. "Estabilizar" el clima: una idea de vital importancia}

¿Cuál es el nivel de perturbación tolerable para el sistema Tierra? Esta pregunta está en el origen de la Convención Marco de las Naciones Unidas sobre el Cambio Climático -que la recoge en su artículo $2-{ }^{16}$, y en torno a ella y a raíz de ella empiezan ${ }^{17}$ los primeros estudios en ciencias humanas, sociales $y$, posteriormente, jurídicos sobre "los límites de la Tierra" (Planetary boudaries) y sobre el Antropoceno.

En esta pregunta clave, a la par científica y política, la Convención Marco encuentra su razón de ser, ya que esta se fija como objetivo último "la estabilización de las concentraciones de gases de efecto invernadero en la atmósfera a un nivel que impida interferencias antropógenas peligrosas en el sistema climático"18. El reto al que se enfrenta la comunidad científica y que se plasma en los textos jurídicos sobre el cambio climático se convierte en una obligación de reduccion ${ }^{19}$. Asimismo, queda claro también que se trata de conseguir estabilizar el nivel de emisiones en la atmósfera de modo que el sistema climático no se vea a su vez perturbado de modo peligroso $^{20}$. Resulta, pues, indispensable adoptar medidas que permitan dicha estabilización, que también debe integrar los parámetros que hagan posible que ningún cambio natural en los ecosistemas se agrave debido a las perturbaciones antrópicas del clima. Por ello, al diseñar las medidas de estabilización del nivel de emisiones $\mathrm{y}$, por consiguiente, del sistema climático, cabe tener en cuenta los cambios naturales de los ecosistemas y sus consecuencias en el sistema climático.

La estabilización del sistema climático implica, según el IPCC, que las emisiones de $\mathrm{CO}_{2}$ no excedan la capacidad de absorción de la biosfera y del océano, y exige una

\footnotetext{
${ }^{16}$ Artículo 2 CMNUCC: "El objetivo último de la presente Convención y de todo instrumento jurídico conexo que adopte la Conferencia de las Partes, es lograr, de conformidad con las disposiciones pertinentes de la Convención, la estabilización de las concentraciones de gases de efecto invernadero en la atmósfera a un nivel que impida interferencias antropógenas peligrosas en el sistema climático. Ese nivel debería lograrse en un plazo suficiente para permitir que los ecosistemas se adapten naturalmente al cambio climático, asegurar que la producción de alimentos no se vea amenazada y permitir que el desarrollo económico prosiga de manera sostenible".

17 Véase sobre esta cuestión, TORRE-SCHAUB, "La construction juridique d'un droit..." cit., pp. 4265.

${ }^{18}$ Artículo 1 de la CMNUCC, sobre las definiciones.

19 "Nuestros cálculos muestran con certeza que: [...] el $\mathrm{CO}_{2}$ es responsable de más de la mitad del aumento del efecto invernadero terrestre; para estabilizar las concentraciones de gases de larga duración en sus niveles actuales se requeriría una reducción de más del $60 \%$ de las actividades humanas que emiten estos gases".

${ }^{20}$ Fórmula utilizada en el artículo 2 CMNUCC.
} 
reducción drástica de las emisiones, que debería implementarse sin demora ${ }^{21}$. Todo esto se contempla ya en el primer informe del IPCC de 199022, así como en la Convención Marco de 1992 y en el segundo informe de 1996. El Protocolo de Kioto de 1997 se aprueba con el fin de implantar los mecanismos que permitan dicha reducción ${ }^{23}$. La cuestión que cabe plantearse en torno a este texto no es, pues, su desconexión respecto a los datos científicos, sino la incapacidad de los responsables políticos para negociar un texto suficientemente ambicioso que permitiera estabilizar las emisiones en el corto período de tiempo aconsejado por la comunidad científica ${ }^{24}$. Y aunque el Protocolo de Kioto fue redactado con cierta ambición y energía, más tarde surgieron algunas dificultades cuando las divergencias políticas lo convirtieron más en un texto de la "división" que en un texto de la "concordia". Trataremos sobre este punto ulteriormente.

21 "Basándonos en los modelos actuales, predecimos: bajo BAU [negocio habitual] un aumento de la temperatura media global durante el [siglo XXI] de aproximadamente $0,3^{\circ} \mathrm{C}$ por década (con un rango de incertidumbre de 0,2 a $0,5^{\circ} \mathrm{C}$ por década); esto es mayor que lo visto en los últimos 10.000 años; bajo el cual [...] asuma niveles cada vez mayores de controles, tasas de aumento en la temperatura media global de aproximadamente $0,2^{\circ} \mathrm{C}$ [a] aproximadamente $0,1{ }^{\circ} \mathrm{C}$ por década".

22 Primer informe del IPCC de 1990 (resumen): "Estamos seguros de lo siguiente: hay un efecto invernadero natural [...]. Concentraciones de $\mathrm{CO}_{2}$, metano, CFC y óxido nitroso. Estos aumentos incrementarán el efecto invernadero, lo que resultará en un calentamiento adicional de la superficie de la Tierra. El principal gas de efecto invernadero, el vapor de agua, aumentará en respuesta al calentamiento global y lo agravará aún más".

${ }^{23}$ El segundo informe de evaluación, Cambio Climático 1995, se presentó en la segunda sesión de la Conferencia de las Partes de la CMNUCC y proporcionó el punto de partida para las negociaciones del Protocolo de Kioto a la CMNUCC. Incluye tres informes de los grupos de trabajo y un resumen de temas científicos y técnicos relacionados con la interpretación del artículo 2 ("Objetivo") de la CMNUCC. En cinco años, la investigación científica ha progresado y el segundo informe del IPCC enfatiza desde el principio que "un conjunto de pruebas sugiere que hay una influencia perceptible del hombre en el clima global". Si bien esta parte antropogénica todavía es difícil de evaluar con precisión, el progreso logrado ahora permite distinguirla de las influencias naturales. La comunidad científica señala que el aumento de las emisiones de gases de efecto invernadero (incluyendo el metano, cuya concentración en la atmósfera se incrementó casi un $145 \%$, y el $\mathrm{CO}_{2}$, con un aumento del $30 \%$ desde la época preindustrial) se debe en gran medida al "uso de combustibles fósiles, cambio de uso de la tierra y agricultura". Algunas tendencias descritas en el informe de 1995 (calentamiento de 0,3 a $0,6{ }^{\circ} \mathrm{C}$ desde finales del siglo XIX, aumento del nivel del mar de 10 a $25 \mathrm{~cm}$ durante un siglo) muestran que un nuevo proceso está en camino y que no va a detenerse.

Los escenarios basados en modelos climáticos contemplan un incremento promedio de la temperatura de entre $1^{\circ} \mathrm{C}$ y $3,5^{\circ} \mathrm{C}$ para el 2100 y un aumento del nivel del mar de 15 a $95 \mathrm{~cm}$. Se espera que el fenómeno del calentamiento global incremente las variaciones generales de temperatura, con un aumento del número de días muy calurosos y una disminución de los días muy fríos. Además, se advierte de las posibles consecuencias de este calentamiento en los diversos ecosistemas del planeta; algunas regiones pueden verse más afectadas por sequías, lluvias torrenciales o inundaciones. Un cambio que finalmente podría modificar "el equilibrio de las especies e incluso conducir a la disminución de los bosques" y, al mismo tiempo, causar una alteración en la cantidad de carbono recogido y emitido por las principales comunidades de plantas y animales del mundo.

${ }^{24}$ DAHAN, A. y AYKUT, S., "Les négociations climatiques : vingt ans d'aveuglement ?", CERISCOPE Environnement, 2014 (en línea). 
Volviendo a la cuestión de la coherencia y relación entre la información científica y los textos de derecho, si bien la conexión entre sistema climático, nivel de emisiones de gases de efecto invernadero a la atmósfera y perturbaciones de los ecosistemas queda claramente establecida desde los primeros informes del IPCC y se plasma también en la Convención Marco de 1992, no puede decirse que en el desarrollo posterior de los demás textos sobre el cambio climático estos factores hayan sido desarrollados de modo preciso ${ }^{25}$. El Protocolo de Kioto, como hemos dicho anteriormente, a pesar de tener una naturaleza obligatoria, se centra en la forma de distribuir las emisiones que cada país puede emitir o intercambiar y no tiene como punto esencial la implantación de un sistema operativo suficientemente "holístico" capaz de conectar la cuestión de la estabilización del clima con la de la estabilización de los ecosistemas. Esta laguna, deplorable, es una de las muchas razones por las que los textos jurídicos sobre el cambio climático no han estado totalmente en la misma línea que los informes científicos del IPCC.

Otro factor que debía considerarse a la hora de diseñar un texto jurídico que tuviera en cuenta los enunciados del IPCC era el de la capacidad de estabilizar las emisiones a la atmósfera en un lapso de tiempo que permitiera mantener la producción de alimentos libre de amenazas (uso de las tierras, sequía, inundaciones) y continuar el desarrollo económico de modo sostenible ${ }^{26}$. Mientras que el segundo objetivo siempre ha estado presente en las negociaciones internacionales sobre el cambio climático, el primero no se contempla en todos los textos. El Protocolo de Kioto no le da suficiente espacio y se centra más en los mecanismos económicos que permiten una distribución de las emisiones por país y por sector, así como en los sistemas de financiación de proyectos de desarrollo y tecnológicos que contribuyan a ese objetivo, más que en una reflexión sobre la seguridad alimentaria, los riesgos de las sequías, la desertificación, la deforestación, etc.

No obstante la dificultad de llegar a un acuerdo internacional capaz de plasmar en obligaciones jurídicas la necesidad de "estabilizar el sistema climático" de modo "regional", se lograron algunas metas. Por ejemplo, la Unión Europea establece a finales de la década de 1990 y principios del milenio el objetivo de que el

${ }^{25}$ DAHAN, A., "L'impasse de la gouvernance climatique globale depuis vingt ans. Pour un autre ordre de gouvernementalité", Critique internationale, núm. 62, 2014, pp. 21-38.

${ }^{26}$ Véase TORRE-SCHAUB, "La construction juridique d'un droit..." cit., p. 45 y ss. 
calentamiento no supere los $2{ }^{\circ} \mathrm{C}$. Y busca reducir las emisiones para el 2050 entre el $50 \%$ y el $85 \%$. Disminución que se deseaba, allá por el año 2008 , que se pudiese continuar igualmente en el siglo XXI, es decir, hoy. Asimismo, según el tercer informe del IPCC del $2006^{27}$, para conseguir esa estabilidad en Europa se necesitaría una disminución de las emisiones de un factor 10. En cambio, las discusiones políticas en esa misma época eran mucho menos ambiciosas, ya que el G8 proponía reducir a la mitad las emisiones hasta el 2050, lo cual comportaría una estabilización del clima en $2,5^{\circ} \mathrm{C}$. De esta forma, se superaría peligrosamente la cifra "mágica" de los $2{ }^{\circ} \mathrm{C}$, que tanto el IPCC como la UE consideran que no se debe sobrepasar. Ante tales diferencias entre lo que "se debería hacer", "lo que se podría hacer" y lo que "finalmente políticamente se hará", ¿cómo podía encontrar un texto jurídico su "lugar" y cómo conseguir que, de forma eficaz y operativa, las propuestas e información científicas del IPCC fuera ratificadas y plasmadas en un texto jurídicamente obligatorio?

Por ello, es importante entender cómo se elaboran los informes del IPCC para comprender mejor la parte de dichos informes que corresponde solo al campo científico y la parte que pertenece a la esfera política, porque los textos jurídicos son el producto de esta última, sin duda, pero contienen elementos que pertenecen claramente al primero.

\section{La epistemología científica del IPCC como método de consolidación normativa}

El segundo elemento que debe ser aquí señalado es el del contenido formal de los informes del IPCC, ya que sus características permitirán también que sea más fácil

\footnotetext{
${ }^{27}$ El tercer informe de evaluación, Cambio climático 2001, también incluye tres informes de los grupos de trabajo: "La base científica", "Impactos, adaptación y vulnerabilidad" (consecuencias, adaptación y vulnerabilidad) y "Mitigación" (medidas de mitigación) -y un informe de síntesis que aborda una amplia gama de temas científicos y técnicos directamente relacionados con las políticas-. En él se han mejorado las proyecciones de los modelos climáticos, y hay más bases de datos y un mejor análisis de estos. En el 2001, el trabajo del grupo I proporciona una imagen más clara del clima global y del fenómeno del calentamiento global en curso. Un calentamiento promedio de aproximadamente $0,6^{\circ} \mathrm{C}$ desde 1861 (fecha de las primeras mediciones) y que es $0,15^{\circ} \mathrm{C}$ más alto que el estimado por los análisis anteriores. Los satélites también aportan información sobre los efectos del cambio climático, mostrando, por ejemplo, que la cubierta de nieve "muy probablemente" ha disminuido en un $10 \%$ desde finales de la década de 1960 y la superficie del hielo marino se ha fundido desde la década de 1950 entre un $10 \%$ y un $15 \%$ durante el verano y la primavera. Asimismo, el nivel medio del mar aumentó de 0,1 a 0,2 metros durante el siglo XX.
} 
poner en marcha el proceso de elaboración de la Convención ${ }^{28}$. Por ello, para una mejor comprensión de la articulación entre información científica y elaboración del derecho del clima en el marco de los textos redactados en las negociaciones de la $\mathrm{ONU}$, es importante entender la forma en la que se redactan estos informes y el subsiguiente proceso de aprobación y discusión.

Algunos datos precisos sobre el contenido de los informes ayudan a entender este proceso de articulación entre ciencia y derecho. Los informes del IPCC están escritos por tres grupos que se complementan ${ }^{29}$. Igualmente, existe para cada uno de los informes de grupo un informe resumido escrito por los autores de cada capítulo y son ellos mismos quienes están a cargo de estos capítulos y también de los grandes informes de cada grupo. Hay además un resumen técnico y, especialmente, un resumen para los responsables políticos. Este resumen en particular se aprueba línea por línea en la reunión de aprobación, que gira en torno a los informes del IPCC. Este es un punto clave, ya que los responsables de las políticas dependen en gran medida de estos informes para liderar las negociaciones sobre el clima. Estas negociaciones dejan paso posteriormente a los textos de la Convención.

Pero ¿cómo se lleva a cabo el proceso de adopción y de qué modo se validan los conocimientos científicos que posteriormente serán utilizados durante las negociaciones y se plasmarán en los textos jurídicos? Los autores del informe del IPCC acuden a la reunión de aprobación con un texto que se discute línea por línea. Todos los países miembros de las Naciones Unidas asisten a esa reunión y discuten el informe para los responsables de políticas con el fin de aprobarlo o modificarlo. Los cambios solo son posibles si lo que propone un gobierno se basa en un argumento cuyo origen se puede identificar en el informe principal. Es decir, solo se podrá modificar el contenido científico de ese informe en virtud de otros datos científicos presentes en el informe principal. Así pues, dado que el informe para los responsables políticos es elaborado por un grupo determinado del IPCC, solo puede ser modificado si se basa y remite a los contenidos científicos de los trabajos de dicho grupo. Dicho de otro modo, el contenido científico que servirá de base a la negociación política de un texto jurídico -la Convención Marco- solo puede

\footnotetext{
${ }^{28}$ Véase LECLERC, "Les règles de production des énoncés..." cit. pp. 11 a 89.

29 Véase supra nota 10.
} 
encontrar su fuente en los propios trabajos del grupo de expertos. Lo cual significa que, al menos en teoría, los textos jurídicos sobre el cambio climático se basan estrictamente en datos proporcionados, revisados y validados por el IPCC.

Así pues, en cuanto al contenido del informe, no hay posibilidad de que un representante de un gobierno pueda cambiarlo. Ello implica, por tanto, que las informaciones científicas cruciales que se refieren al cambio climático -aumento de la temperatura, probabilidades de incremento del nivel del mar, deshielo de los glaciares...- deben obtenerse del proprio trabajo del IPCC y que los responsables políticos no pueden añadir ni alterar dichos datos. El resumen para los responsables políticos que deben tomar las decisiones refleja los principales informes.

Así pues, aunque la elaboración del derecho climático es un mecanismo altamente politizado, el proceso de adopción del resumen para los responsables políticos es el punto fuerte de los informes del IPCC, puesto que, una vez que se aprueba, los funcionarios de cada gobierno encargados de negociar los textos jurídicos solo pueden incluir en ellos aquello que ya se recoja en el informe del IPCC. En algunos países, las mismas partes participan en la aprobación de los informes del IPCC y representan a sus países en la negociación de la Convención sobre el Clima.

El Protocolo de Kioto tenía, por una parte, la finalidad de continuar con el objetivo de "estabilización del clima" y, por otra, conseguir que los Estados participaran activamente en las políticas de reducción y mitigación del fenómeno del calentamiento global. Por ello, pese a sus defectos, debe ser visto como un primer paso en la voluntad de la comunidad internacional de disminuir el nivel de emisiones con respecto a 1990 (año del primer informe del IPCC). Aunque hoy sabemos que el Protocolo no tuvo el éxito esperado, ya que algunos países nunca llegaron a ratificarlo, es importante comprender su planteamiento para entender mejor cómo se desarrolló el diálogo entre ciencia y derecho en este período.

\section{Lenguaje científico y lenguaje jurídico: ¿una dinámica económica?}

Mientras que el primer informe del IPCC inspira y contribuye al inicio del proceso de negociación de la Convención Marco, así como de las sucesivas conferencias de la partes, el segundo informe, de junio de 1996, permite el acceso a una serie de informaciones cruciales para la puesta en marcha de la Convención. Este informe 
constituye la antesala del futuro Protocolo de Kioto de 1997, ya que en mayo de 1996 el IPCC publica su Metodología para el inventario nacional de emisiones de gases de efecto invernadero. Este inventario permite, por una parte, establecer una metodología común para todos los Estados parte en la Convención Marco. Y, por otra, posibilita que los Estados dispongan de información precisa sobre sus trayectorias de gases de efecto invernadero con el fin de que adopten sus respectivas estrategias de reducción en el marco de las políticas de mitigación. Asimismo, el resumen para los responsables políticos de 1995, la síntesis del segundo informe — publicada igualmente en 1995- y el informe del grupo III del IPCC, sobre las consecuencias económicas y sociales del cambio climático publicado en julio de 1995-, sirven de guía a los diferentes países para poder comenzar, durante la COP que tuvo lugar ese mismo año en Bonn, a establecer las bases del futuro Protocolo de Kioto ${ }^{30}$. Términos que hasta entonces no se habían utilizado en el lenguaje jurídico, como mitigación, vulnerabilidad o trayectoria, empiezan a formar parte del lenguaje jurídico. Del mismo modo, el informe publicado en 1997, relativo a las vulnerabilidades regionales, permite que, sobre la base del Protocolo de Kioto ya negociado, se fije una estrategia de ayuda a los países más vulnerables, tanto desde el punto de vista geográfico como económico, con la puesta en funcionamiento de los proyectos de ayuda al desarrollo de nuevas tecnologías.

El Protocolo de Kioto de 1997, que logra ver la luz gracias a todo ese conjunto de informes generales y parciales del IPCC publicados entre 1992 y 1997, tiene en cuenta para su operatividad una serie de cuestiones económicas y desarrolla diversos instrumentos jurídicos y de mercado cuya finalidad es implantar un sistema de distribución universal y global tanto de responsabilidades como de permisos de emisión. De esta forma, en respuesta a los informes científicos del IPCC, la comunidad internacional apuesta por los instrumentos económicos y de mercado como herramienta jurídica de operatividad del Protocolo ${ }^{31}$.

En el segundo informe del IPCC la comunidad científica dice por primera vez que existe "un conjunto de elementos que sugieren una influencia perceptible del hombre

\footnotetext{
30 TORRE-SCHAUB, "La protection juridique du climat..." cit., p. 51 y ss.

31 TORRE-SCHAUB, "La protection juridique du climat..." cit., p. 54 y ss.; Decision 27/CMP, Procedimientos y mecanismos relativos al respeto de la disposiciones del Protocolo de Kioto, Anexo XV, punto 5, FCCC/KP/CMP/2005/8/Add.3.
} 
en el clima"32. A partir de esta idea, se empieza a pensar que el hombre es la causa y el origen del cambio climático, el denominado "origen antropógeno"33. Y políticas como las del vicepresidente de los Estados Unidos Al Gore contribuyen a la implementación del Protocolo de Kioto. Resulta evidente que, de no haber sido por esa frase del segundo informe del IPCC, habría sido muy difícil poner en marcha el funcionamiento operativo de la Convención Marco que se plasma en el Protocolo de Kioto.

Dicho Protocolo, recordémoslo, es de naturaleza claramente económica, ya que establece los llamados "instrumentos de mercado": los mercados de permisos de emisión de gases de efecto invernadero, los proyectos de desarrollo, etc. Sin embargo, y a pesar de este marcado carácter económico, este texto pone en evidencia de nuevo, siguiendo la dinámica empezada en Río cuando se redactó la Convención Marco, la crucial importancia de la información científica a la hora de elaborar textos jurídicos sobre el cambio climático.

\section{El Protocolo de Kioto: ¿dónde queda la "universalidad" de la lucha contra el}

\section{cambio climático?}

La negociación del instrumento jurídico encarnado por el Protocolo de Kioto estuvo marcada desde el principio por la separación del mundo en dos polos: Norte y Sur, países ricos y países pobres. A esta división — que, por otro lado, tenía su lógica-, se añadió la consiguiente diferenciación de responsabilidades. De esta forma, en este instrumento se recoge el principio, ya enunciado en Río por la Convención Marco de 1992, de las "responsabilidades comunes pero diferenciadas"34. Una vez elaborado y firmado dicho instrumento, los países también debían pensar en la segunda fase del Protocolo: el período del 2008 al 2012 35 . La consolidación de un régimen jurídico climático que incluía el objetivo de reducción del total de emisiones de los países del Norte de "al menos" un 5\% con respecto a 1990 en el período del

\footnotetext{
32 Véase supra nota 20.

33 Véase supra nota 20.

34 BARTENSTEIN, K., "De Stockholm à Copenhague: genèse et évolution des responsabilités communes mais différenciées dans le droit international de l'environnement", McGill Law Journal, $56: 1,2010$, p. 182 ; MICHELOT, A., "Le principe des responsabilités communes mais différenciées", RJE, Dossier spécial RIO+20, núm. 4, 2012, p. 633.

35 MALJEAN-DUBOIS, A., "Les suites de kyoto. Les post 2012 : quelles perspectives pour le régime juridique international de lutte contre le réchauffement climatique ?", Torre-Schaub (dir.), Dossier Spécial Droit... cit., pp. 111-125.
} 
2008 al 2012 (artículo 3, párrafo 1 del Protocolo) sentó las bases para el inicio de la reflexión en torno a la negociación y el diseño de un nuevo instrumento jurídico que sustituyera al Protocolo. Es decir, ya en el 2008 se empezaba a pensar en el post 2015, cuando se haría necesario un nuevo instrumento, puesto que el Protocolo tenía fecha de caducidad y no podía prolongarse más allá del 2018.

El universalismo que había caracterizado la Convención Marco, y que había sido igualmente la esperanza de muchos durante la negociación del Protocolo de Kioto, se vio ya desde la entrada en vigor del Protocolo, en el 2005, dificultado por la división económica y geográfica entre Norte y Sur. Esta división conllevaba no solo que las responsabilidades fueran distintas entre ambos grupos, sino también la cantidad de emisiones que cada país de esos dos grupos debía reducir. La postura de algunos de los países del Norte (Estados Unidos, Canadá), que, aun asumiendo su responsabilidad de tener que reducir más y más rápido, no aceptaban que los países del Sur estuvieran en su mayoría exentos de cualquier responsabilidad, dinamitó el régimen del Protocolo y provocó su prematura condena a muerte. Los citados países del hemisferio Norte presionaron políticamente a la comunidad internacional para que algunos países del Sur -Brasil, China e India- fueran excluidos del régimen de exención de responsabilidad del que gozaban los restantes países del Sur con el fin de que esos tres países contribuyeran también a la política de reducción como si fueran países desarrollados. Este "muro de China" ${ }^{36}$ que los Estados Unidos levantaron a principios de la década del 2000 provocó que el Protocolo no cumpliera su función y que no fuera posible implantar un régimen jurídico de distribución y reducción de $\mathrm{CO}_{2}$ eficaz y operativo a escala universal.

El régimen jurídico del Protocolo de Kioto, a pesar de haber sido pensado para ser obligatorio, nunca llegó a serlo del todo, por lo que los objetivos científicos que lo habían impulsado en su origen quedaron sepultados tanto por unos instrumentos económicos que nunca cumplieron su función como por diferencias políticas y económicas que iban mucho más allá de la lucha contra el cambio climático e incluían problemas de rivalidad y competencia comercial e industrial entre los Estados Unidos y China. El régimen climático se vio influido por razones ajenas a la ciencia y el derecho y se convirtió en el caballo de Troya de una lucha comercial e

\footnotetext{
36 VIÑUALES, J., "The Paris Agreement, un initial examination", Cambridge University, Working Papers C-EENRG, núm. 6, 2015 https://papers.ssrn.com/sol3/papers.cfm?abstract id=2704670
} 
industrial entre dos potencias económicas rivales. Por ello era necesario e imperativo pensar en un nuevo régimen jurídico para el post 2012 que fuera capaz de volver a reconectar la ciencia con el derecho, los instrumentos jurídicos con la voluntad política de la comunidad internacional y de cada Estado.

Junto con estos elementos, la sociedad civil, entendida en un sentido amplio ciudades, empresas, ONG-, comenzó a hacerse un hueco en las negociaciones y en los eventos paralelos que acompañaban a las conferencias de la partes (side events), y consiguió que sus reivindicaciones empezaran a ser más oídas. Esto, sumado al hecho de que los nuevos informes del IPCC, el cuarto y quinto, mostraban que el calentamiento era ya irreversible y que no solo existía el riesgo de desestabilización del sistema climático sino también del sistema económico y político de nuestras sociedades, motivó que la comunidad internacional se reuniera aún más frecuentemente con el fin de diseñar un nuevo instrumento jurídico capaz de superar las carencias del Protocolo de Kioto e integrar los nuevos parámetros científicos de urgencia y a los nuevos actores emergentes en el juego político de la lucha contra el cambio climático ${ }^{37}$.

\section{EL ACUERDO DE PARÍS Y LA RELACIÓN ENTRE CIENCIA Y DERECHO, ¿LOST IN TRANSLATION?}

Partiendo de la necesidad de reencontrar la filosofía de un acuerdo universal capaz de hacer revivir el espíritu de la Convención Marco y de superar los problemas que habían surgido con el Protocolo de Kioto, la comunidad internacional tenía ante sí un reto importante. En cuanto al rol del derecho, era obvio que resultaba de vital importancia que sirviera para reconciliar la comunidad internacional, muy dividida por las diferencias económicas, comerciales e industriales que el Protocolo había acentuado e incluso potenciado. Era importante, pues, que el derecho internacional pudiese de nuevo cumplir el papel que le había sido confiado en 1992 en Río durante la Cumbre de la Tierra: garantizar a escala mundial la lucha contra el calentamiento global. Las diferencias económicas provocadas por el Protocolo de Kioto, demasiado dependientes de las ventajas y desventajas comparativas, y alimentadas por una omnipresente lógica económica, debían solucionarse mediante

${ }^{37}$ TORRE-SCHAUB, "Vers un nouveau paradigme..." cit., p. 42 y ss. 
otro instrumento jurídico. Ello no implicaba el abandono total de los instrumentos de mercado, ya que estos pueden resultar atractivos para implicar al sector privado en la reducción de las emisiones, pero era más necesario que nunca tener en cuenta todos estos factores para poder reflexionar de forma madura y responsable en torno a un futuro marco jurídico del régimen climático internacional. El derecho estaba más que nunca llamado a jugar su papel de herramienta de reconciliación entre la ciencia y la política, la política y la economía y los principios de equidad y responsabilidad, que siempre han estado muy presentes en la cuestión del calentamiento global.

Partiendo de estos elementos, se empieza a negociar el post Kioto y el futuro instrumento jurídico que debía reemplazarlo.

Antes de abordar las diferentes etapas que permitieron llegar al Acuerdo de París, es necesario recordar el papel jugado por la información científica en las negociaciones de este instrumento y el lugar que ocupa hoy, en el momento de implantación del Acuerdo. Nuestro hilo conductor en esta parte será, pues, doble, al igual que en la primera: seguiremos un cierto orden cronológico para comprender mejor las etapas de la creación del régimen jurídico climático, pero tendremos en cuenta también las informaciones científicas de los informes del IPCC que han ido acompañando este proceso.

Dado que ya en el 2008 -apenas tres años después de la entrada en vigor del Protocolo de Kioto y cuatro años antes del 2012, final del segundo período del Protocolo- se sabía que nunca se alcanzaría el objetivo de "estabilizar el clima" y que las trayectorias de reducción con respecto al nivel de emisiones de 1990 indicadas por el Protocolo no conducirían a ese objetivo, se decidió preparar el período post 2012 con suficiente antelación. Varios elementos debían tenerse en cuenta a la hora de establecer un nuevo instrumento jurídico para el régimen climático: alcanzar el objetivo de la estabilización del clima, lograr la reducción y estabilización de las emisiones para el 2050 y aprobar un nuevo instrumento jurídico "universal" capaz de unir a todos los países en vez de separarlos en bloques enfrentados como había hecho - muy a su pesar- el Protocolo de Kioto.

Dado que el régimen instaurado por el Protocolo finalizaba en el 2012, urgía acordar un régimen post Kioto — denominado por algunos Kioto II- antes de esa fecha. Las razones tanto económicas como ecológicas eran ya evidentes en el 2008, cuando se empieza a pensar en el nuevo régimen. La pregunta, no obstante, que surgía sin 
cesar era: ¿debe establecerse un instrumento que dé continuidad a Kioto o más bien otro que lo sustituya?

Puesto que el objetivo de estabilización en torno a los $2{ }^{\circ} \mathrm{C}$ no era discutible ni negociable, lo que debía negociarse antes de ser plasmado en un texto jurídico era la manera de conseguir ese objetivo. En otras palabras, qué naturaleza jurídica debía revestir el nuevo instrumento del régimen climático de modo que se pudieran respetar los objetivos científicos de urgencia marcados por el IPCC sin volver a caer en los errores políticos y económicos de Kioto, teniendo en cuenta el imperativo de elaborar un instrumento "universal" e "inclusivo" tanto a nivel geográfico como político, económico y social. ¿Cómo poner sobre el papel de modo coherente el imperativo científico recogido en el informe del IPCC del 2006 y su complemento del 2007 y el retraso hasta ahora sufrido en las políticas de reducción tanto a nivel nacional como mundial?

\section{Irreversibilidad, mitigación y adaptación, ¿qué instrumento jurídico?}

El nuevo instrumento jurídico que debería cubrir el período 2012-2050 tendría ante todo que cumplir la condición apuntada en el informe del IPCC del 2007 de incluir a todos los países emisores de gases de efecto invernadero, independientemente de si son países del hemisferio Norte o Sur. Era, pues, importante adoptar ese punto de vista. En Bali, en el 2007, la Conferencia de las Partes, que contó con una participación masiva de países y ONG, preveía en su orden del día la adopción de un acuerdo global $^{38}$. En Copenhague, en el 2009, también se contempló ese objetivo, pero no se llegó a ningún resultado. Se necesitaban acciones ambiciosas tanto en materia de mitigación como de adaptación, ya que los informes del IPCC entre el 2007 y el 2009 señalaban la urgencia no solo de reducir las emisiones, sino también y, dado el carácter avanzado del calentamiento global y su inevitable irreversibilidad, de tomar medidas de adaptación para aquellas regiones que corren ya un alto riesgo de ser víctimas del cambio climático.

38 GUÉRIN, E., "L'accord de Copenhague : que s'est-il passé ? Est-ce un bon accord ? Qui y gagne et qui y perd ? Et maintenant ?", IDDRI, Doc de travail, 2009, https://www.iddri.org/fr/publications-etevenements/document-de-travail/laccord-de-copenhague-que-sest-il-passe-est-ce-un (consultado el 26/04/2019). 
Con la cuestión de la adaptación surge también de forma urgente la problemática de la financiación de estas medidas. ¿Cómo ayudar a las víctimas del cambio climático? ¿Cómo contribuir a financiar la adaptación en aquellos países que se verán más afectados por los efectos negativos del calentamiento global?

Se adopta así un método de participación extensiva con un marco multilateral laxo, de modo que los Estados Unidos acepten formar parte del nuevo instrumento jurídico y los pequeños Estados insulares y emergentes puedan integrarse en el régimen climático como plenos actores y no como participantes relegados al anexo $\mathrm{B}$, como era el caso con el régimen de Kioto.

La postura americana fue en esa ocasión más flexible que en otros casos. Empezando por el hecho de que aceptaron la legitimidad de los informes del IPCC y accedieron a sentarse a la mesa con todos los países en pie de igualdad. Así, a finales del 2007 en Bali, los Estados Unidos están de nuevo en la mesa. No obstante, sus condiciones de aceptación de un régimen obligatorio e universal climático no agradan a todas las partes. Para ellos, en primer lugar es indispensable que todos los países, incluso aquellos en vías de desarrollo, participen en las políticas de reducción de emisiones ${ }^{39}$. En segundo lugar, se oponen a que una instancia central marque los objetivos de disminución y consideran que cada país debe decidir sus propios objetivos. Los Estados Unidos abogan, pues, ya desde el 2008, por elaborar el nuevo texto climático desde una perspectiva bottom up y abandonar el método top down, el utilizado para Kioto y la Convención.

Así pues, en Bali, de forma discreta pero muy influyente, se produce un cambio no solo en la estrategia de perspectiva y reflexión sobre el nuevo instrumento, sino en el lenguaje mismo que se utiliza a partir de entonces por voluntad de los Estados Unidos: se pasa de hablar de países del anexo I y anexo II a hablar de países "desarrollados" y países "en vías de desarrollo". Esta flexibilidad, sutil pero persistente desde entonces, abrió una nueva era en las negociaciones climáticas, ya que los países debían participar en el régimen universal climático en función de su nivel de desarrollo actual y no en el de 1990. Esto tiene su relevancia en cuanto a

39 GUÉRIN, E. y WEMAERE, M., "The Copenhagen Accord: What happened? Is it a good deal? Who wins and who loses? What is next?", CLIMATE, núm. 08/09 diciembre de 2009 www.iddri.org. 
las consignas de alcanzar un objetivo de reducción para no superar los $2{ }^{\circ} \mathrm{C}$ aconsejados por el IPCC.

Este cambio notable marcó el futuro del nuevo instrumento y allanó el camino hasta el Acuerdo de París del 2015. Hubo también diversas dificultades que debían ser salvadas o al menos discutidas. Una cuestión de gran relevancia fue la siguiente: si bien resultaba claro que los objetivos marcados por el nuevo texto debían perseguir la "estabilidad del clima", ¿debían ser objetivos de resultados —con plazos a respetar pero con total libertad respecto a los medios empleados - u objetivos de medidas concretas a implantar — políticas energéticas, instrumentos de mercado, fiscalidad de carbono, incentivos a las empresas reductoras de emisiones, etc.-? Es decir, qué naturaleza jurídica debía tener el nuevo instrumento que se negociaba. Este fue uno de los puntos de mayor clivage en las negociaciones y retardó la elaboración y la votación de un nuevo texto. La urgencia señalada por el IPCC en sus últimos informes, que consideraban indispensable acordar un texto post Kioto antes de diciembre del 2012, no se tuvo en cuenta y no fue hasta el 2015 , tras tres años de duras discusiones y diferencias en las conferencias de las partes, que se llegó a un acuerdo. $\mathrm{Y}$, si bien el objetivo de la "estabilización en $2{ }^{\circ} \mathrm{C}$ " no se abandona nunca sobre el papel, la imposibilidad de traducirlo en términos jurídicos suficientemente inclusivos y obligatorios hace muy difícil poder alcanzar dicho objetivo.

El plan de acción propuesto en el 2008, a pesar de no llegar a ser un instrumento jurídico, consiguió mantener el objetivo del IPCC y tener en cuenta el contenido del tercer informe, que era el de incluir las "políticas de reforestación y de gestión durable de los bosques y selvas" en las políticas de mitigación y adaptación. De este modo se empezaron a diseñar unos nuevos parámetros, que se retomarán en el Acuerdo de París, consistentes en proponer políticas de incentivos para el sector privado a fin de que participe en la reducción de emisiones mediante la compensación con políticas forestales y ayudas financieras, ya sea en el propio país o en países en los que desarrolle planes de mitigación o de nuevas tecnologías (equivalentes al europeo REDD y REDD+).

La inclusión del sector financiero en el nuevo instrumento jurídico que se negocia se acepta unánimemente y no se discute que el régimen jurídico del clima debe tener en cuenta las finanzas en su texto. No cabe ya la posibilidad de conseguir los 
objetivos de reducción y adaptación sin contar con los actores financieros. Ello, además, va a dar seguridad a los países que aún se muestran reticentes a la idea de firmar un acuerdo universal. Asimismo, se crean varios fondos de financiación de la adaptación y de pérdidas y daños.

A pesar de estos avances, resulta muy difícil ponerse de acuerdo sobre unas bases comunes y operativas de participación. Algunas divergencias se refieren al hecho de decidir si el diseño de las políticas debe corresponder solo a los Estados y Gobiernos o si hay que incluir también a las empresas. También plantearon dudas cuestiones como las ayudas financieras o la voluntariedad u obligatoriedad de las políticas. Todos estos temas ocuparon varias conferencias y reuniones, sin que se lograra llegar a un consenso satisfactorio para todos.

\section{Copenhague, ¿una línea discontinua en el diálogo entre ciencia y derecho?}

El Acuerdo de Copenhague, que debía diseñar un régimen global sobre el clima post 2012, resulta muy decepcionante desde el punto de vista jurídico y científico. Este acuerdo, considerado "blando", no solo no lleva a ningún compromiso sobre un instrumento jurídicamente obligatorio para el post Kioto, sino que potencia, de facto, el aumento del calentamiento mucho más allá del límite de $2^{\circ} \mathrm{C}$ puesto por el IPCC desde 1990.

En efecto, el Acuerdo de Copenhague implica que las partes solo están obligadas por las decisiones tomadas dentro de la COP de conformidad con las reglas de procedimiento (de aplicación provisional), artículo 8 de la Convención Marco, dado que el Acuerdo se alcanzó informalmente en los márgenes de la COP. El Acuerdo de Copenhague debe ser considerado como una decisión política que solo expresa una intención de actuar desde varios países bien identificados (que se enumeran en el preámbulo del Acuerdo).

Se expresa simplemente que la Convención debe ser implementada con el fin de lograr su objetivo final, pero no se dice cómo.

El enfoque bottom up para establecer objetivos de resultados es muy inconsistente e incoherente entre las metas a largo plazo y aquellas a corto plazo. Todo ello conduce a los países más vulnerables, como las pequeñas islas del Pacífico, hacia un aumento de $3{ }^{\circ} \mathrm{C}$ o incluso de $3,5^{\circ} \mathrm{C}$, poniendo su mera existencia en un riesgo 
muy alto ${ }^{40}$. Sin embargo, las Maldivas aceptaron el Acuerdo porque se les propuso, a cambio de su voto favorable, una interesante financiación de la adaptación al cambio climático.

Los países africanos, al igual que los pequeños Estados insulares, también se arriesgaban a un aumento mínimo de $3^{\circ} \mathrm{C} \circ 3,5^{\circ} \mathrm{C}$, lo que se traduciría en un incremento real de más de $5{ }^{\circ} \mathrm{C}$ en caso de seguir las políticas propuestas por el Acuerdo. Esto alejaba considerablemente la trayectoria de calentamiento global de los $2{ }^{\circ} \mathrm{C}$ que el IPCC postula en sus diferentes informes y que la Convención Marco y el Protocolo de Kioto habían asumido. Sin embargo, casi todos estos países africanos respaldaron la declaración del Acuerdo de Copenhague, probablemente debido también a la posibilidad de recibir ayuda financiera para su adaptación.

Tampoco había ninguna referencia a un futuro instrumento legalmente vinculante en el futuro. $Y$ el enfoque bottom up y voluntario de las contribuciones individuales de cada país no se traducía en un $50 \%$ de reducción de las emisiones globales para el 2050, tal y como los informes del IPCC habían aconsejado. La sección dedicada a la adaptación era igualmente muy floja, y las medidas de respuesta se ponían en pie de igualdad con los impactos del clima. El Acuerdo tampoco recogía ningún objetivo cuantificado para reducir las emisiones provocadas por la deforestación y la degradación de los bosques.

El texto adoptado (parcialmente) no solo no era un acuerdo universal - que era lo deseable para cumplir las sugerencias del IPCC-, sino que era muy poco ambicioso y no permitiría de ninguna manera logar los objetivos de disminución de emisiones para evitar un aumento global de las temperaturas para el 2050. Dada la total discontinuidad, por no decir ruptura, con los consejos de los expertos del IPCC, se necesitaba de forma urgente superar la crisis sufrida en Copenhague y movilizar a la comunidad internacional en una llamada de SOS para conseguir sustituir el Protocolo de Kioto para el período siguiente -aun cambiando los instrumentos jurídicos empleados - con un nuevo acuerdo suficientemente obligatorio y ambicioso como para poder "estabilizar" la temperatura en torno a los $2{ }^{\circ} \mathrm{C}$.

40 GUÉRIN y WEMAERE, "The Copenhagen Accord...", cit. 


\section{3. "Siempre nos quedará París...": el Acuerdo de París, una continuidad aparente}

Las conclusiones del informe del IPCC del 2014 eran muy claras y ello permite decir que existe una continuidad entre dicho informe y la Conferencia de París del 2015, que llevó al Acuerdo de París, hoy vigente.

Uno de los puntos fuertes del quinto informe del IPCC es que distingue entre lo que es humano y lo que eventualmente es de origen natural ${ }^{41}$. Dicho informe demostró que gran parte del calentamiento global de los últimos cincuenta años está casi seguramente relacionado con actividades humanas. Al contrario de los informes previos, que avanzaban esta hipótesis con mayor o menor grado de incertidumbre, el quinto informe del IPCC sostuvo este vínculo ${ }^{42}$. Ello conllevó que las Partes negociadoras de la Convención Marco se convencieran de la insuficiencia del Acuerdo de Copenhague y de la absoluta necesidad de adoptar un nuevo acuerdo que abarcara todos los Estados del planeta (acuerdo universal) y que asumiera de modo indiscutible, desde el preámbulo, la urgencia y necesidad de limitar la temperatura a $2^{\circ} \mathrm{C}$ como máximo.

El Acuerdo de París es presentado a menudo como el primer acuerdo universal sobre el clima. Pretende ser diferenciado, justo, sostenible, dinámico, equilibrado y legalmente vinculante ${ }^{43}$. Fue aprobado por las 195 delegaciones el 12 de diciembre del 2015 y entró en vigor el 4 de noviembre del 2016. El 7 de noviembre del 2017, tras la adición de la firma siria, 196 de los 197 países de las Naciones Unidas firmaron o se comprometieron a firmar el Acuerdo de París, lo que lo convierte en el acuerdo más extendido y el más rápidamente firmado en la historia de la humanidad. Al margen del Acuerdo, el Desafío de Bonn ha sido ratificado y ampliado con el objetivo de reforestar 350 millones de hectáreas de tierra degradada o deforestada

\footnotetext{
${ }^{41}$ El quinto informe del IPCC presenta varios desarrollos nuevos en términos de metodología o atribución de responsabilidades por fenómenos climáticos. También reafirma que el aumento de las concentraciones de gases de efecto invernadero podría comportar cambios importantes en la temperatura, el nivel del mar o el derretimiento del hielo. La conclusión es muy clara: las actividades humanas, especialmente el uso de combustibles fósiles, han producido un aumento excepcional en la concentración de gases de efecto invernadero que transforman el clima a una velocidad nunca antes vista.

42 "El vínculo entre las actividades humanas y el aumento de las temperaturas es extremadamente probable $(+95 \%$ de probabilidad)". Informe del Grupo I, elementos científicos, https://leclimatchange.fr/les-elements-scientifiques/ (consultado el 28/04/2019).

${ }^{43}$ BOISSON DE CHAZOURNES, L., "Regards sur l'Accord de Paris -Un Accord qui batit le futur-", Torre-Schaub, M. (dir.), Bilan et perspectives de l'Accord de Paris (COP 21), regards croisés, Colection Institut Tunc, Publications de l'IRJS, París, 2017, p. 97 y ss.
} 
para el 2030, lo que supondrá otro modo de retomar lo establecido en el último informe del IPCC.

El Acuerdo plantea contener para el 2100 el calentamiento global "muy por debajo de $2{ }^{\circ} \mathrm{C}$ en comparación con los niveles preindustriales" y, si es posible, "continuar los esfuerzos para limitar el aumento de las temperaturas a $1,5{ }^{\circ} \mathrm{C}$ ", (artículo 2), lo cual es más ambicioso que el proyecto de acuerdo original. Este último objetivo se añadió por la presión de la Alianza de los Pequeños Estados Insulares (AOSIS), que reúne a los 44 países más expuestos a los efectos del cambio climático (los cuales, por otro lado, son los que emiten la menor cantidad de gases del mundo: 0,00001\% de las emisiones globales. No obstante, el texto de la decisión observa "con preocupación que los niveles estimados de las emisiones agregadas de gases de efecto invernadero en 2025 y 2030 resultantes de las contribuciones previstas determinadas a nivel nacional no son compatibles con los escenarios de $2{ }^{\circ} \mathrm{C}$ de menor costo sino que conducen a un nivel proyectado de 55 gigatoneladas en 2030, y observa también que, para mantener el aumento de la temperatura media mundial por debajo de $2{ }^{\circ} \mathrm{C}$ con respecto a los niveles preindustriales, mediante una reducción de las emisiones a 40 gigatoneladas, o por debajo de $1,5{ }^{\circ} \mathrm{C}$ con respecto a los niveles preindustriales [...] se requerirá un esfuerzo de reducción de las emisiones mucho mayor que el que suponen las contribuciones previstas determinadas a nivel nacional”44.

El artículo 2 también se refiere a la desinversión de combustibles fósiles: "El presente Acuerdo [...] apunta a fortalecer la respuesta mundial a la amenaza del cambio climático, [...] en particular por [...] compatible con un perfil de desarrollo hacia un desarrollo con bajas emisiones de gases de efecto invernadero y resiliencia al cambio climático".

El objetivo de alcanzar la neutralidad de carbono se establece en el artículo 4: "[...] las Partes se proponen lograr que las emisiones mundiales de gases de efecto invernadero alcancen su punto máximo lo antes posible [...] y a partir de ese momento reducir rápidamente las emisiones de gases de efecto invernadero [...] para alcanzar un equilibrio entre las emisiones antropógenas por las fuentes y la absorción antropógena por los sumideros en la segunda mitad del siglo". Esto se

${ }^{44}$ Artículo 2 del Acuerdo, https://unfccc.int/sites/default/files/french paris agreement.pdf (consultado el 27/04/2019). 
denomina "emisión neta cero": reduciendo nuestras emisiones de GEI para que, en la segunda mitad del siglo, sean compensadas por los sumideros de carbono (bosques, océanos, técnicas de captura y almacenamiento de carbono).

Al recordar el principio de "responsabilidades comunes pero diferenciadas" de la Convención Marco de 1992, el Acuerdo exige que "los países desarrollados continúen liderando el camino asumiendo los objetivos de reducción de emisiones en términos absolutos". Los países en desarrollo "deben continuar aumentando sus esfuerzos de mitigación [...] con respecto a diferentes contextos nacionales"45. Se establece así una diferenciación entre los países más industrializados y los países en desarrollo.

El techo de la ayuda climática para los países en desarrollo se ha fijado en 100.000 millones de dólares (91.000 millones de euros) por año y será revisado por 2025 a más tardar. Los objetivos anunciados a nivel nacional se revisarán para el 2020 y luego cada cinco años, y los objetivos de reducción de emisiones solo podrán revisarse al alza (artículo 4.3). Se realizará una evaluación general del Acuerdo en el 2023 y luego cada cinco años. Sin embargo, este ciclo de revisión está sujeto a cambios decididos en el marco de las COP posteriores.

Pese a los elementos positivos expuestos, el Acuerdo presenta numerosas lagunas y defectos que ponen en duda su eficacia y, por tanto, el cumplimiento del objetivo de la estabilización en torno a los $2^{\circ} \mathrm{C}$. Por ejemplo, el abandono de la extracción de combustibles fósiles, considerado como un elemento importante de la transición climática, no se menciona. Arabia Saudí se opuso desde un principio, y con éxito, a cualquier mención de las palabras combustibles fósiles, petróleo o carbón. Sin embargo, es bien sabido que los combustibles fósiles son la principal causa del cambio climático y representan el $65 \%$ de todas las emisiones de GEI. Si se quiere mantener el calentamiento por debajo de los $2{ }^{\circ} \mathrm{C}$, el $80 \%$ de las reservas fósiles se deberían quedar en el subsuelo. Igualmente, todas las referencias a las emisiones de gases de efecto invernadero de la aviación y el transporte marítimo internacional han desaparecido de la versión final del texto. Sin embargo, representan el $8 \%$ de las emisiones globales totales y están creciendo tan rápido (el doble que en otros

45 Preámbulo del Acuerdo, punto 2 de la Decisión, https://unfccc.int/sites/default/files/french paris agreement.pdf (consultado el 27/04/2019). 
sectores) que, si no se hace nada, podrían representar el 39\% de las emisiones para el 2050. La Organización Marítima Internacional todavía no muestra ninguna voluntad de cambiar su política en favor del medio ambiente. Peor aún, la Asociación de Transporte y Medio Ambiente confía más bien poco en la capacidad de la Organización de Aviación Civil Internacional y la Organización Marítima Internacional para reducir las emisiones aéreas y marítimas y, por lo tanto, respetar el Acuerdo de París. En general, tampoco se abordan los problemas demográficos en el Acuerdo -a pesar de que aparecen en los trabajos del grupo III del quinto informe del IPCC— ni se ha considerado ninguna medida de control de la natalidad.

El objetivo de "continuar los esfuerzos para limitar el aumento de las temperaturas a $1,5^{\circ} \mathrm{C}$ " parece poco probable, puesto que en el 2015 la temperatura promedio del planeta ya estaba cerca de $1^{\circ} \mathrm{C}$ por encima del nivel preindustrial. Además, el potencial de calentamiento de los gases de efecto invernadero que ya se encuentran en la atmósfera es de $0,3^{\circ} \mathrm{C}$ en comparación con el nivel actual y las partículas finas reflectantes, que enfrían el clima en $0,3{ }^{\circ} \mathrm{C}$, están destinadas a desaparecer con el uso de tecnologías menos contaminantes. Por lo tanto, aunque todas las emisiones cesaran de inmediato, la temperatura global podría continuar aumentando en aproximadamente $0,6^{\circ} \mathrm{C}$, posiblemente superando el objetivo de $1,5^{\circ} \mathrm{C}$.

Sin embargo, el proyecto ADVANCE ${ }^{46}$, realizado durante tres años por catorce institutos de investigación europeos para desarrollar una nueva generación de escenarios, concluyó en octubre del 2016 que el objetivo de limitar el aumento de la temperatura a $1,5{ }^{\circ} \mathrm{C}$ era técnicamente posible, si bien implicaría medidas draconianas que aún no han empezado a tomarse. Si se espera que las emisiones netas de gases de efecto invernadero se reduzcan a cero para el 2050 y caigan por debajo de cero durante la segunda mitad del siglo $\mathrm{XXI}$, ello requeriría una dependencia masiva de las tecnologías de extracción de dióxido de carbono (eliminación de dióxido de carbono, como el secuestro de dióxido de carbono), que se espera que eliminen la mitad de las 1.000 Gt de $\mathrm{CO}_{2}$ que se emitirían del 2011 al 2100 en estos escenarios. Estas técnicas, no obstante, conllevan altos riesgos y plantean numerosas incertidumbres en cuanto a su seguridad, datos alarmantes que, de hecho, el propio IPCC ha puesto de manifiesto en su quinto informe y que

\footnotetext{
${ }^{46}$ ADVANCE desarrolló una nueva generación de modelos avanzados de evaluación integrada y métodos aplicados para explorar diferentes opciones de políticas de mitigación del clima en el marco posterior a París. El proyecto finalizó en diciembre del 2016, http://www.fp7-advance.eu/
} 
subraya con especial fuerza en su último informe especial, El mundo a $1,5{ }^{\circ} \mathrm{C}$, publicado el 8 de octubre del $2018^{47}$.

Un estudio publicado el 31 de julio del 2017 en la revista Nature Climate Change estimaba en un $5 \%$ la probabilidad de limitar el calentamiento global a $2{ }^{\circ} \mathrm{C}$ para el 2100, que es, sin embargo, el objetivo establecido por el Acuerdo de París. Las posibilidades de alcanzar la meta de $1,5^{\circ} \mathrm{C}$, también recogida en el texto del Acuerdo, son solo del 1\%, pronóstico que incluye esfuerzos para limitar el uso de combustibles fósiles y estima un aumento de la temperatura para el 2100 entre $2{ }^{\circ} \mathrm{C}$ y $4,9^{\circ} \mathrm{C}$, con un valor promedio de $3,2^{\circ} \mathrm{C}^{48}$.

Aunque el Acuerdo de París supone un relativo avance - con respecto al de Copenhague - y retoma una relación fuerte entre ciencia y derecho, su propia puesta en funcionamiento puede provocar un retroceso en dicha relación. En efecto, si bien la Conferencia de París de la COP 21 de diciembre del 2015 que dio lugar al del Acuerdo de París se basó claramente en el quinto informe del IPCC, actualmente podemos observar un riesgo preocupante de distanciamiento entre la ciencia y el derecho. Ello se explica por varios motivos. Por una parte, el carácter "voluntario" y bottom up del Acuerdo, a pesar de ser universal. Por otra, el hecho de que en las últimas conferencias de las partes no ha habido progresos en cuanto al aumento de la ambición de los objetivos de reducción. A ello se suman los problemas derivados de la falta de ayudas financieras a los países emergentes para la toma de medidas de mitigación eficaces. Asimismo, la coyuntura internacional es muy poco prometedora: los Gobiernos de los Estados Unidos y de Brasil ponen en peligro la propia supervivencia del Acuerdo. Igualmente, hay que tener en cuenta el problema de los países que dependen totalmente del carbón y las energías fósiles, como Canadá, los Estados Unidos, Rusia, Arabia Saudí y, en Europa, Polonia. A ello debe añadirse la "tibia" acogida que ha tenido el último informe especial del IPCC del 8 de octubre del 2018 durante la Conferencia de la Partes (COP 24) en Katowice,

\footnotetext{
47 Uno de los mensajes clave de este informe especial es la idea de que "cada medio grado cuenta". Las consecuencias, ya desastrosas a $1,5^{\circ} \mathrm{C}$, son mucho más graves a $2{ }^{\circ} \mathrm{C}$. Además, estudios recientes sugieren que, por encima de este umbral, el aumento de las temperaturas podría provocar efectos dominó, principalmente irreversibles, relacionados con el derretimiento del hielo y el permafrost, el cambio en la circulación oceánica... Estos efectos comportarían cambios mucho más drásticos que los previstos actualmente, transformando el medio ambiente más allá de lo que ha observado la humanidad. Limitar el calentamiento al nivel más bajo posible puede ser incluso más vital de lo que sugiere el informe.

$48 \mathrm{http} / / / \mathrm{www} . \mathrm{fp} 7-$ advance.eu/?q=news/nature-climate-change-publishes-advance-results-sensitivitylong-term-co2-emissions
} 
Polonia, informe que fue solo "anotado", y no "altamente acogido", como era de esperar $^{49}$. Esta poco generosa y fría acogida del informe - a pesar de que fue explícitamente pedido tras la COP 21 de París, en febrero del 2016, con el fin de "tener más información sobre los peligros de una estabilización en torno a $2{ }^{\circ} \mathrm{C}$ en vez de en torno a $1,5^{\circ} \mathrm{C}$ "- puede poner en peligro el futuro del Acuerdo e, incluso peor, animar a los países a no alinearse con los objetivos del Acuerdo marcados para el 2050 — la neutralidad de carbono_. Lo cual equivaldría a firmar la sentencia de muerte de todo el proceso de construcción de un régimen jurídico climático iniciado hace ya más de cuarenta años- capaz de protegernos contra el calentamiento global ya inevitable y contra las consecuencias negativas imparables que se avecinan.

\section{CONCLUSIÓN}

Por todo lo dicho anteriormente, debemos concluir con una nota optimista y con otra más prudente en cuanto al futuro de la relación entre ciencia y derecho en la construcción del régimen jurídico del clima. Si bien es cierto que los informes del IPCC y de algunos otros grupos de expertos, como la NAS en los Estados Unidos y diversos institutos de investigación científica europeos (CNRS, Grantham Institute, IPSL), han contribuido en gran medida a la elaboración del Acuerdo de París, su implementación — su cumplimiento, seguimiento y desarrollo futuro-corre el riesgo de crear cierta "discontinuidad" en la relación entre ciencia y derecho. Esto debe ser visto como algo preocupante, ya que la ciencia climática es la que hasta ahora ha permitido movilizar a la comunidad internacional en torno al peligro que representa el calentamiento global. Así pues, es importante que las futuras negociaciones internacionales en torno al seguimiento y funcionamiento del Acuerdo de París

\footnotetext{
${ }^{49} \mathrm{El}$ informe describe la viabilidad técnica y teórica de limitar las trayectorias del calentamiento de 1,5 ${ }^{\circ} \mathrm{C}$, y propone acciones para implementar este objetivo. En este contexto, reducir las emisiones mucho antes del 2030 es una condición esencial. Los autores enfatizan, por una parte, la absoluta urgencia de la acción y, por otra, las dificultades para implementar tecnologías nunca utilizadas a una escala tan grande. Además, el desarrollo descontrolado de estas tecnologías puede entrar en conflicto con la preservación de la biodiversidad o el desarrollo de las poblaciones. No basta con pensar que el desarrollo y la innovación contrarrestarán las emisiones de las actividades humanas. Para alcanzar el objetivo de limitación de $1,5^{\circ} \mathrm{C}$, se necesita una reducción drástica e inmediata de las emisiones y un avance tecnológico nunca visto en esta escala. Sin embargo, la posibilidad de observar estos dos fenómenos en los próximos años está disminuyendo cada día desde el primer informe del IPCC de 1988. Como recordatorio, cabe señalar que actualmente estamos avanzando hacia un calentamiento de $3{ }^{\circ} \mathrm{C}$ para finales de siglo. Si y solo si se respetan los compromisos del Acuerdo de París. https://www.ipcc.ch/sr15/, (consultado el 26/04/2019).
} 
tengan más en cuenta los informes del IPCC, sobre todo el último informe especial sobre los $1,5^{\circ} \mathrm{C}$. Dicho documento es el más claro y contundente en cuanto a la necesidad de adoptar medidas urgentes para tener una mínima posibilidad de lograr la estabilidad del clima en torno a los $2{ }^{\circ} \mathrm{C}^{50}$. A pesar de que el informe fue casi ignorado en la última COP en Polonia —debido a la clara oposición de los países más dependientes de las energías fósiles_-, es de vital importancia retomarlo en la próxima COP 25, que tendrá lugar en Chile en diciembre del 2019, e incluirlo de alguna forma en la implementación del Acuerdo de París y en la nueva fase de revisión del Acuerdo prevista para el 2023, ya que el futuro y la supervivencia del sistema Tierra y de la especie humana están en peligro. El último informe del IPCC del 8 de octubre pasado es categórico en este sentido: "[...] si queremos conseguir estabilizar el clima, debemos tomar ya las medidas más drásticas y sólidas posibles en cuanto a la reducción de emisiones y en cuanto al desarrollo de políticas de adaptación y de justicia social y económica, de modo que sea posible acompañar a las poblaciones más vulnerables en el proceso de supervivencia y adaptación al calentamiento que ya está en marcha y que se agravará y acelerará considerablemente en los años venideros" ${ }^{51}$.

\section{BIBLIOGRAFÍA}

BARSTENSTEIN K., "De Stockholm à Copenhague: genèse et évolution des responsabilités communes mais différenciées dans le droit international de l'environnement", McGill Law Journal, 56:1,2010, p. 182.

BOISSON DE CHAZOURNES, L., "Le droit et l'universalité de la lutte contre le changement climatique", Torre-Schaub, M. (dir.), Dossier Spécial Droit et Climat, pp. 29-37.

\footnotetext{
50 Una limitación del calentamiento a $1,5^{\circ} \mathrm{C}$ reduciría significativamente los riesgos para los sistemas naturales y humanos en comparación con un aumento de las temperaturas a $2{ }^{\circ} \mathrm{C}$ (el objetivo optimista más popular en la actualidad). Los compromisos asumidos en virtud del Acuerdo de París son claramente insuficientes para limitar el calentamiento a $1,5^{\circ} \mathrm{C}$. Los compromisos nacionales actuales nos llevan por un camino de $+3^{\circ} \mathrm{C}$ para finales de siglo y un calentamiento que continúa más allá, https://www.ipcc.ch/sr15/, (consultado el 26/04/2019).

51 Véanse los trabajos de WAISMAN, H., "Quelle base scientifique pour la justice climatique", TorreSchaub, M. y Lormeteau, B. (dirs.), Les recours climatiques, Influences et convergences de la décision Urgenda et du Rapport spécial du GIEC à $1,5^{\circ} \mathrm{C}$, Revue Energie, environnement, Infrastructures, Dossier spécial, mayo del 2019, pp. 15-18; y TORRE-SCHAUB, M., "Quelle efficacité pour les recours climatiques en France : apports de la décision Urgenda et du Rapport du GIEC à $1,5^{\circ} \mathrm{C}$, Torre-Schaub y Lormeteau (dirs.), Les recours climatiques... cit., pp. 25-30.
} 
— "Regards sur l'Accord de Paris -Un Accord qui batit le futur", Torre-Schaub, M. (dir.), Bilan et perspectives de l'Accord de Paris (COP 21), regards croisés, Colection Institut Tunc, Publications de I'IRJS, París, 2017, p. 97 y s.

DAHAN, A. y AYKUT, S., "Les négociations climatiques : vingt ans d'aveuglement ?", CERISCOPE Environnement, 2014.

DAHAN, A. y GUILLEMOT, H., "Les relations entre science et politique dans le régime climatique : à la recherche d'un nouveau modèle d'expertise ?", Natures, Sciences, Sociétés, vol. 23, núm. Supplément, S6 - S 18, 2015.

DAHAN, A., "L'impasse de la gouvernance climatique globale depuis vingt ans. Pour un autre ordre de gouvernementalité”, Critique internationale, núm. 62, 2014, pp. 21 38.

— “La gouvernance climatique : entre climatisation du monde et schisme de réalité", L'Homme et la Société, núm. 199, pp. 79-90, julio del 2016.

Decisión 27/CMP, Procedimientos y mecanismos relativos al respeto de la disposiciones del Protocolo de Kioto, Anexo XV, punto 5, FCCC/KP/CMP/2005/8/Add.3.

GUERIN, E. y WEMAERE, M., "The Copenhagen Accord: What happened? Is it a good deal? Who wins and who loses? What is next?", Climate, núm. 08/09, diciembre del 2009; www.iddri.org

GUERIN, E., "L'accord de Copenhague : que s'est-il passé ? Est-ce un bon accord ? Qui y gagne et qui y perd ? Et maintenant ?", IDDRI, Document de travail, 2009, https://www.iddri.org/fr/publications-et-evenements/document-de-travail/laccord-decopenhague-que-sest-il-passe-est-ce-un

IPCC, informes, sobre todo el de 1995, Les Cahiers de Global Chance, núm. 7, julio 1996.

JOUZEL, J., "La pluridisciplinarité au cœur du problème”, Torre-Schaub, M. (dir.), Dossier Spécial Droit et Climat, Les Cahiers Droit, Sciences \& Technologies, núm. 2, 2009, pp. 19-29.

—_La pluridisciplinarité au cœur du problème”, Torre-Schaub, M. (dir.), Dossier Spécial Droit et Climat, Les Cahiers Droit, Sciences \& Technologies, p. 20. 
LECLERC, O., "Les règles de production des énoncés au sein du Groupe d'experts gouvernmental sur l'évolution du climat", Encinas de Munagorri, R. (dir.), Expertise et gouvernance du changement climatique, LGDJ, coll Droit et Société, París, 2009, pp. 59-89.

MALJEAN-DUBOIS, A., "Les suites de Kyoto. Les post 2012 : quelles perspectives pour le régime juridique international de lutte contre le réchauffement climatique ?", Torre-Schaub, M. (dir.), Dossier spécial Droit et Climat, Les Cahiers Droit, Sciences \& Technologies, 2009, pp. 111-125.

MICHELOT, A., "Le principe des responsabilités communes mais différenciées", RJE, Dossier spécial RIO+20, núm. 4, 2012, p. 633.

NAIM-GISBERT, E., Les dimensions scientifiques du droit de l'environnement, Contribution à l'étude des rapports science-droit, Bruylant, 1999.

SINAI, A., "Le climat, otage des lobbies industriels", Le Monde diplomatique, febrero 2001, p. 14.

TORRE- SCHAUB M., "La construction juridique d'un droit à un climat stable : le droit autour de $2^{\circ} \mathrm{C}$ ", Normes et Changement climatique, regards interdisciplinaires, Actes du Colloque du 9 novembre 2018, Lancement du GDR ClimaLex, Torre-Schaub, M. et al. (dirs.), Mare et Martin, París, 2019, pp. 42-65.

- "La protection juridique du climat, entre gestion contractuelle et négociation économique", Revista Themis, Revista da Facultade de Direito da UNL, IV-núm. 6, 2003, pp. 47-41.

- "Le réchauffement climatique, une question interdisciplinaire ", introduction, TorreSchaub, M. (dir.), Dossier Spécial Droit et Climat, Les Cahiers Droit, Sciences \& Technologies, núm. 2, 2009, pp. 13-19.

- "Vers un nouveau paradigme socio-environnemental dans la gouvernance du climat", Torre-Schaub, M., Lavorel, S. y Moliner-Dubost, M. (dirs.), Quel(s) $\operatorname{droit}(s)$ pour les changements climatiques ?, Mare et Martin, París, 2018, pp. 35-55 y 42.

VIÑUALES, J., "The Paris Agreement, un initial examination", Cambridge University, Working Papers C-EENRG, núm. 6, diciembre 2015 https://papers.ssrn.com/sol3/papers.cfm?abstract id=2704670 
WAISMAN, H., "Quelle efficacité pour les recours climatiques en France : apports de la décision Urgenda et du Rapport du GIEC à $1,5^{\circ} \mathrm{C}$ ", Torre-Schaub, M. y Lormeteau, B. (dirs.), Les recours climatiques, Influences et convergences de la décision Urgenda et du Rapport spécial du GIEC à $1,5^{\circ} \mathrm{C}$ Revue Energie, environnement, Infrastructures, Dossier spécial, mayo 2019, pp. 25-30.

WAISMAN, H., "Quelle base scientifique pour la justice climatique", Torre-Schaub, M. y Lormeteau, B. (dirs.), Les recours climatiques, Influences et convergences de la décision Urgenda et du Rapport spécial du GIEC à $1,5^{\circ} \mathrm{C}$, Revue Energie, environnement, Infrastructures, Dossier spécial, mayo 2019, pp. 15-18. 